$\begin{array}{ll}\text { Research Square } & \begin{array}{l}\text { Preprints are preliminary reports that have not undergone peer review. } \\ \text { They should not be considered conclusive, used to inform clinical practice, } \\ \text { or referenced by the media as validated information. }\end{array}\end{array}$

\title{
Infection within 2 weeks before liver transplantation closely related to prognosis of posttransplant infection: a single-center retrospective observational study in China
}

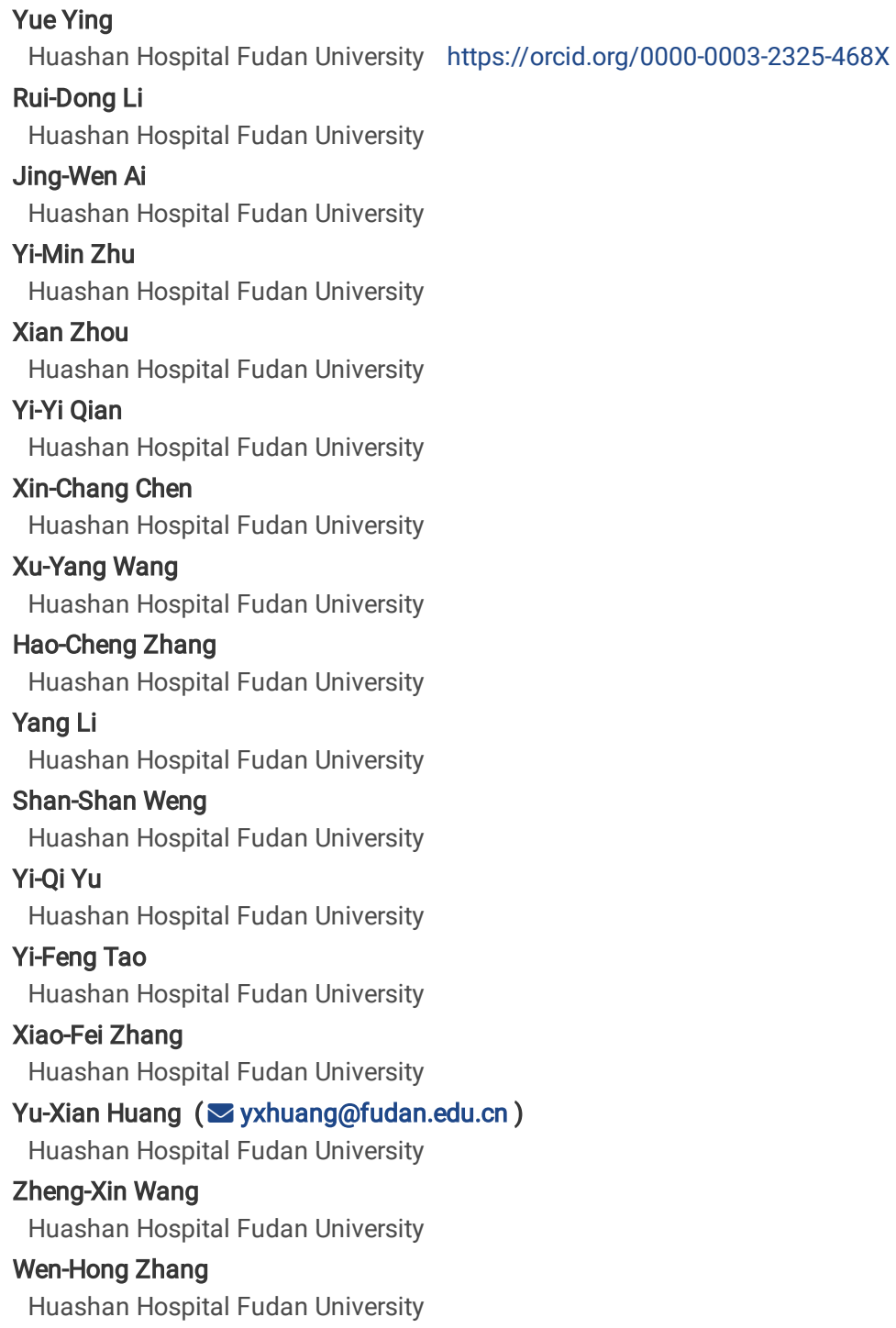

Research article

Keywords: Infection; liver transplantation; epidemiology; risk factors; MELD score

Posted Date: May 17th, 2019

DOI: https://doi.org/10.21203/rs.2.9647/v1

License: () (7) This work is licensed under a Creative Commons Attribution 4.0 International License. Read Full License

Version of Record: A version of this preprint was published at Hepatobiliary \& Pancreatic Diseases International on August 1st, 2020. See the published version at https://doi.org/10.1016/j.hbpd.2020.06.001. 


\section{Abstract}

Background Infections still represent the main factors influencing morbidity and mortality following liver transplantation. This study is to evaluate the incidence and risk factors for infection and survival after liver transplantation. Methods We retrospectively examined medical records in 210 recipients who underwent liver transplantation between April 2015 and October 2017 in our center. Results During the median follow-up days of 214 , the incidence of infection after liver transplantation was $46.7 \%(n=98)$ : namely, pneumonia (43.4\%), biliary tract infection (21.9\%) and peritonitis (21.4\%). Among the pathogens in pneumonia, the most frequently isolated was Acinetobacter baumanii (23.5\%) and Klebsiella pneumoniae (21.1\%). For biliary tract infection, the first rank was Strenotrophomonas maltophilia (14.0\%) and then Klebsiella pneumoniae (11.6\%). Pseudomonas aeruginosa, Strenotrophomonas maltophilia, and Klebsiella pneumoniae accounted for $21.4 \%, 11.9 \%$ and $11.9 \%$ of pathogens in peritonitis, respectively. The independent risk factors for infection after liver transplantation are model for end-stage liver disease (MELD) or pediatric end-stage liver disease (PELD) score, total blood loss in operation and duration of drainage tube. All-cause mortality was $11.0 \%(n=23)$. The prognostic factors for postoperative infection in transplant recipients are infection, especially pneumonia within 2 weeks before transplantation, complication with impaired renal function and higher MELD or PELD score after 7 days of transplantation. Kaplan-Meier curves of survival showed that recipients with infection within 2 weeks before transplantation had a significantly lower cumulative survival rate compared with those without infection $(66.7 \%$ vs $91.9 \%, \mathrm{HR}=4.480,95 \% \mathrm{Cl}, 3.377-47.85 ; \mathrm{p}<0.001)$. Conclusions Infection, especially pneumonia within 2 weeks before transplantation are independent prognostic factors for postoperative infection in transplant recipients.

\section{Background}

Liver transplantation remains an effective procedure for multiple end-stage liver diseases. With the widespread use of potent immunosuppressive agents, the incidence of allograft rejection was reduced while opportunistic infections increased. Despite efficacious antimicrobial prophylaxis and vaccination to some pathogens, infections still represent the main factors influencing morbidity and mortality following liver transplantation. ${ }^{1-5}$

In infections after liver transplantation, epidemiologic exposures included donor-derived infections and recipient-derived infections. Transplanted organs facilitate the transmission of infections from organ donors. ${ }^{1}$ Colonized or latent infections of recipients could be activated due to immunosuppression state after transplantation. However, the data about these were rare.

According to several previous reports, the spectrum of major infections occurring after transplantation has undergone a striking evolution ${ }^{4}$. So, knowledge of pathogen spectrum was very useful to help effective empirical treatment. In this study, we surveyed infection rate and pathogen distribution after liver transplantation and analyzed the impact of donor or recipient factors on infection and survival.

\section{Methods}

\section{General population}

This was a retrospective, single center research. A total of 217 consecutive patients who underwent liver transplantation between April 2015 and October 2017 were eligible to participate in the research. 7 patients were excluded because they died within 7 days after transplantation. At last, 210 patients were included in the study. Patient records were reviewed at the end of the study and follow-up information was obtained from referring physicians. Surveillance viral load testing for cytomegalovirus (CMV) were performed every week for the first month after transplant. Other cultures were ordered as dictated by the clinical situation. The study was approved by the hospital's ethics committee.

\section{Surgery and immunosuppression}

All patients underwent orthotopic liver transplantation either with primary anastomosis of the bile duct or choledochojejunostomy.

Standard immunosuppression often included tacrolimus (FK506), mycophenolate (MMF) and corticosteroids. FK506 was initially administered at a dosage of $3 \mathrm{mg}$ every 12 hours orally as soon as the patient was able to take oral medication, usually less than three days after surgery. FK506 was then adjusted according to blood levels. The target level for tacrolimus was 10-12 ng/ml during the first month and 8-10 $\mathrm{ng} / \mathrm{ml}$ after the second month. Oral MMF was given at the dosage of $500 \mathrm{mg}$ twice a day after 12 days of surgery. $240 \mathrm{mg}$ of methylprednisolone was administered intravenously on the day of surgery. This was reduced to $180 \mathrm{mg}$ on the day after surgery and then rapidly tapered so that by day 6 the patient was receiving 40 mg of methylprednisolone. Oral therapy with an equivalent dose of prednisone was employed when the patient was able to take oral medicine. Baxilimab (20mg) was given intravenously in the surgery as dictated by clinical situation.

\section{Antimicrobial treatment}

Once recipients were diagnosed as infection, they would receive empirical antimicrobial treatment, then turning to etiological treatment after the results of culture and susceptibility were applicable. When viral load testing for CMV was positive, intravenous ganciclovir $250 \mathrm{mg}$ every 12 hours was given until viral eradication was achieved, but not shorter than 2 weeks.

\section{Definition of infections}

We attempted to identify all infections which occurred after transplantation and categorize them according to time after transplantation, site of infection, type of pathogen, and outcome. Strict criteria were employed to define infections included in this study. They are as follows: 
Bloodstream infection was defined by the isolation in at least 1 blood culture of Listeria monocytogenes, Staphylococcus aureus, Candida species or aerobic gram-negative rods. For other pathogens, the isolation of the bacteria in 2 blood cultures or in 1 positive blood culture and in a culture from a known site of infection was required.

Abdominal infection was diagnosed if the peritoneal neutrophil count was $>200$ polymorphonuclear cells and if a pathogen was isolated. In cases where no cell counts were obtained, a Gram stain of peritoneal fluid showing $\geq 1$ polymorphonuclear cell per oil field was sufficient.

Biliary tract infection required the presence of fever, right upper quadrant pain, and elevation of liver function tests together with either evidence of cholangitis on liver biopsy or isolation of the same organism from both the T-tube drain and blood.

Pneumonia required the appearance of new infiltrates on chest radiograph, the onset of new respiratory symptoms (cough, dyspnea) or hypoxemia, and the isolation of bacteria in heavy growth from purulent sputum. A diagnosis of $\mathrm{CMV}$ pneumonia also required a temperature elevation $\geq 38^{\circ} \mathrm{C}$ for at least 2 days within a 4-day period, the presence of neutropenia or thrombocytopenia, and the detection of CMV DNA in blood.

\section{Statistical analysis}

Univariate and multivariate analysis were used to find the risk factors and prognostic factors for infection after transplantation. We used Mann-Whitney $U$ test and chi-square test for the univariate analysis and all variables with a $\mathrm{p}$ value $<0.05$ were defined as significant. We further conducted logistic regression for the multivariate analysis with factors proved to be significant. The Kaplan-Meier method was used to calculate the overall survival in the recipients who got infection after transplantation. All analyses were performed using Statistical Package for the Social Sciences, version 17.0 (SPSS Inc., Chicago, IL) and Graphpad Prism, version 6.02 (Graphpad Software, San Diego, CA, USA).

\section{Results}

\section{Spectrum of patient information and incidence of postoperative infection}

Of all the 210 patients in this study, the most common underlying disease was viral hepatitis ( 139 cases, $66.2 \%)$, then inherited liver diseases ( 24 cases, $11.4 \%$ ) and autoimmune liver disease (16 cases, $6 \%$ ) (Table 1). In total, 196 episodes of infection occurred after transplantation during the median following days of 214. Every patient experienced on average 0.93 episodes of infection. In all, 98 patients were defined as postoperative infection, and the incidence of infection reached $46.7 \%$. (Figure 1 )

The most frequent pathogen was Enterobacteriaceae with 41 episodes, including Klebsiella pneumoniae, Enterobacter cloacae and Serratia marcescens. The second most common isolated organism was Acinetobacter baumanii (27 cases), which only occurred in 1 month after transplantation. Pseudomonas aeruginosa and Strenotrophomonas maltophilia was isolated in 21 and 22 cases, respectively. Regarding viral infection, cytomegalovirus was the most common with 12 episodes.

In total, 12 episodes of fungal infection were noted, and the most frequent pathogen was Candida albicans (10 cases). (Table 2)

\section{Sites of infection and microbial aetiology}

Respiratory tract was the most common site of infection (43.4\%), then biliary tract (21.9\%), abdomen (21.4\%) and bloodstream (7.6\%). Among the pathogen in pneumonia, the most frequently isolated was Acinetobacter baumanii (23.5\%) and Klebsiella pneumoniae (21.1\%). Pseudomonas aeruginosa, Strenotrophomonas maltophilia, and Klebsiella pneumoniae accounted for $21.4 \%, 11.9 \%$ and $11.9 \%$ of pathogens in peritonitis, respectively. For biliary tract infection, the first rank was Strenotrophomonas maltophilia (14\%), then Klebsiella pneumoniae (11.6\%), while Pseudomonas aeruginosa and Enterococcus faecium (9.3\%) were in the equal third place. Only 15 episodes of bloodstream infection occurred, and Staphylococcus was the most common pathogen. (40\%, Figure 2)

\section{Risk factors for infection after liver transplantation}

In univariate analysis, we found infection-related factors included: age, model for end-stage liver disease (MELD) or pediatric end-stage liver disease (PELD) score and infection within 2 weeks before transplantation, especially pneumonia other than peritonitis, amount of blood loss and duration time of drainage tube (Table 3). In multivariate analysis, the independent risk factors of infection after liver transplantation are MELD or PELD before transplantation (18.2 vs $12.4, p=0.002)$, amount of blood loss in operation ( $2.3 \mathrm{~L}$ vs $1.1 \mathrm{~L}, \mathrm{p}=0.002)$ and duration time of drainage tube $(29.6 \mathrm{~d}$ vs $12.8 \mathrm{~d}, \mathrm{p}=0.009)$.

\section{Recipient survival and prognostic factors for infection after liver transplantation}

During the median follow-up days of 214 , all-cause mortality was $11.0 \%(23 / 210)$. The causes of death included three main categories: infection, poor graft function and others. $42 \%$ was infection-associated death, while poor graft function accounted for $29 \%$. In a total of 98 patients with infection after transplantation, 82 people survived. (Figure 1) In the univariate analysis, infection within 2 weeks before transplantation (especially pneumonia other than peritonitis), estimated glomerular filtration rate (eGFR) $<50 \mathrm{ml} / \mathrm{min}$ after transplantation and MELD or PELD score before or after 7 days of transplantation 
were identified as prognostic factors (Table 4). Multivariate analysis revealed that independent prognostic factors were infection within 2 weeks before transplantation (OR 5.3, 95\% Cl, 1.143-24.578) and MELD or PELD score after 7 days of transplantation (OR 1.298, 95\%Cl, 1.105-1.526, Table 3). When recipients suffered from infection within 2 weeks before transplantation, although infection was controlled on the day of transplantation, they had a significantly lower survival compared to those not infected in Kaplan-Meier analysis $(67.6 \%$ vs $91.3 \%, \mathrm{HR}=4.480,95 \% \mathrm{Cl}, 3.377-47.85$; $\mathrm{p}<0.001, \mathrm{Figure} 3)$.

\section{Discussion}

Among all the liver transplant recipients, infection is a problem to be concerned. Comparing to patients with normal immune function, the diagnosis of infection is delayed because diminished signs and symptoms. Also, the spectrum of potential pathogens is broader and infection often progress more rapidly ${ }^{1}$.

So, it is very important to learn the risk and prognostic factors of infection after liver transplantation. In our study, MELD or PELD score is the major risk and prognostic factor regarding to infection after transplantation, which is similar to other studies. ${ }^{6-8}$. MELD or PELD score are used to evaluate the liver function of patients waiting to process liver transplantation, either in adult or pediatric. ${ }^{9-10}$ With extreme poor hepatic function, these recipients might have a lower level of immune defense against invasive microbial. We also found whether MELD or PELD score recover to normal after transplantation is associated with survival rate, like other studies reported ${ }^{11-12}$. With failed recovery of liver function after liver transplantation, patients suffering from infections are more likely to be not survived.

In our hospital, the incidence of infection after liver transplantation reached $46.7 \%$. It was consistent with the previous reports studied in China, in which $15 \%-69 \%$ of patients acquired postoperative infection ${ }^{13-17}$. Most infection occurred in the first month after liver transplantation, which is related to surgical complications as for traditional view ${ }^{18}$. However, the most common site of infection is not surgical site, but the respiratory tract and Gram-negative pathogens are major pathogens. Our findings were also supported by other studies in China ${ }^{14-15}$. These indicated that the pattern of posttransplant infection was changed and nosocomial infection might be the most important epidemiological exposure in the first month after transplantation in China.

The reason for most Chinese patients receiving liver transplantation is cirrhosis based on chronic hepatitis $\mathrm{B}^{19}$. As we found in the study, viral hepatitis accounts for $65.3 \%$ of underlying disease. Spontaneous bacterial peritonitis is a common complication of cirrhosis. Meanwhile, pneumonia and biliary tract infection are also not rare in the case of chronic cirrhosis. As a result, a large number of patients got infection before transplantation.

It is recommended that active infection in transplant recipients should be controlled before transplantation, since immunosuppression will exacerbate the infectious process. ${ }^{1}$ In this study, there were 18 patients (8.6\%) having infections within 2 weeks before transplantation. Although their infections were all controlled on the day of transplantation, it remained to be a risk and prognostic factor for infection after transplantation. Among all the infections, pneumonia seemed to be more related than other infections. In 20 patients suffering pneumonia within 2 weeks before transplantation, 15 patients (75\%) got pneumonia again after transplantation. While among 244 patients without pneumonia before, only $98(40.2 \%)$ got pneumonia after transplantation. $(p=0.004$, data not shown). We assume the explanation might be that the pathogens become latent, aren

teradicatedth or oughlydue $\rightarrow$ lackoftreatmentcourse and causep $\infty$ rerīier function, especiallyf or theres $\pi$ ra $\rightarrow$ rytract, $\leq$ ad $\in$ t prove these infections are exactly recipient-derived, infections within 2 weeks before transplantation should be paid close attention.

This study had some limitations. First, this is a single center study, and the results can be biased because of different pathogen distribution and higher antibiotic resistance rate than other medical institutions in China. Second, our study was retrospective, and the lack of some information, such as bile complication information other than infection, might cause some biases in data statistics.

\section{Conclusions}

We concluded that risk factors for infection after liver transplantation are MELD or PELD score and infection, especially pneumonia within 2 weeks before transplantation. The prognostic factors for postoperative infection in transplant recipients are infection, especially pneumonia within 2 weeks before transplantation, complication with impaired renal function and MELD or PELD score after 7 days of transplantation.

\section{Declarations}

\section{Ethics approval and consent to participate}

The study was approved by the ethics committee of Huashan hospital, Fudan University. Written informed consent was obtained from all participants.

\section{Consent for publication}

Not applicable.

\section{Availability of data and materials}

The datasets used and/or analysed during the current study are available from the corresponding author on reasonable request.

\section{Competing interests}

The authors declare that they have no competing interests. 
Funding

Not applicable.

\section{Author's contributions}

Y.Y participated in the performance of the research, in the writing of the paper, and in data analysis. R.D.L participated in the performance of the research and in the admission and management of the patients. J.W.A participated in data analysis. Y.M.Z, X.Z, Y.Y.Q, X.C.C, X.Y.W, H.C.Z, Y.L and S.S.W participated in the data collection. T.G. participated in general supervision of infection control service and in the performance of the research. Y.Q.Y participated in research design and data analysis. Y.F.T and X.F.Z. participated as general supervision of transplant unit and in the performance of the research. W.H.Z. participated in the revising the article. Y.X.H. and Z.X.W. participated in research design, in the performance of the research and in the writing of the article.

\section{Acknowledgements}

Not applicable.

\section{References}

[1] Fishman J. Infection in solid-organ transplant recipients. N Eng J Med. 2007;357(25):2601-14.

[2] Romero FA and Razonable RR. Infections in liver transplant recipients. World J Hepatol. 2011;3(4):83-92.

[3] Patel R and Paya CV. Infections in solid-organ transplant recipients. Clin Microbiol Rev. 1997; 10(1):86-124.

[4] Chang FY, Singh N, Gayowski T, et al. Fever in liver transplant recipients: changing spectrum of etiologic agents. Clin Infect Dis. 1998;26(1):59-65.

[5] Kawecki D, Chmura A, Pacholczyk M, et al. Bacterial infections in the early period after liver transplantation: etiological agents and their susceptibility. Med Sci Monit. 2009;15(12):CR628-37.

[6] Yoshizumi T, Shirabe K, Ikegami T, et al. Decreased immunoglobulin G levels after living-donor liver transplantation is a risk factor for bacterial infection and sepsis. Transpl Infect Dis. 2014;16(2):225-31.

[7] Avkan-Oguz V, Ozkardesler S, Unek T, et al. Risk factors for early bacterial infections in liver transplantation. Transplant Proc. 2013;45(3):993-7.

[8] Kaido T, Egawa H, Tsuji H, et al. In-hospital mortality in adult recipients of living donor liver transplantation: experience of 576 consecutive cases at a single center. Liver Transplant. 2009;15: 1420-25.

[9] Singal AK and Kamath PS. Model for End-stage Liver Disease. J Clin Exp Hepatol. 2013;3(1):50-60.

[10] Bourdeaux C, Tri TT, Gras J, et al. PELD score and posttransplant outcome in pediatric liver transplantation: a retrospective study of 100 recipients. Transplantation. 2005;79 (9):1273-76.

[11] Györi GP, Silberhumer GR, Rahmel A, de Vries E, Soliman T, Zehetmayer, et al. Impact of dynamic changes in MELD score on survival after liver transplantation - a Eurotransplant registry analysis. Liver Int. 2016;36(7):1011-7.

[12] Zakareya T, Abbasy M, Abdel-Razek W, Elsiesy H, Abal Khail F, Al Sebayel M, et al. Utility of post-liver transplantation MELD and delta MELD in predicting early and late mortality. Eur J Gastroenterol Hepatol. 2017;29(12):1424-27.

[13] Jeong S, Wang X, Wan P, et al. Risk factors and survival outcomes of biliary complications after adult-to-adult living donor liver transplantation. United European Gastroenterol J. 2017;5(7):997-1006.

[14] Li C, Wen TF, Mi K, et al. Analysis of infections in the first 3-month after living donor liver transplantation. World J Gastroenterol. 2012;18(16):1975-80.

[15] Tu Z, Xiang P, Xu X, et al. DCD liver transplant infection: experience from a single centre in China. Int J Clin Pract. 2016;70(Suppl 185):3-10.

[16] Zhang ML, Xu J, Zhang W, et al. Microbial epidemiology and risk factors of infections in recipients after DCD liver transplantation. Int J Clin Pract. 2016;70(Suppl 185):17-21.

[17] Wan QQ, Ye QF, Ming YZ, et al. The risk factors for mortality in deceased donor liver transplant recipients with bloodstream infections. Transplant Proc. 2013;45(1):305-7.

[18] Patel R and Paya C V. Infections in solid-organ transplant recipients. Clin Microbiol Rev. 1997,10(1):86-124.

\section{Tables}

Table 1 Recipient characteristics 


\begin{tabular}{lc}
\hline & Liver transplantation recipients $(\mathrm{n}=210)$ \\
\hline Age, mean (range) & $45.1(0.6-69)$ \\
Gender (Male), n (\%) & $167(79.5)$ \\
Underlying disease & \\
Viral hepatitis, n (\%) & $139(66.2)$ \\
Inherited disease, n (\%) & $24(11.4)$ \\
Autoimmune liver disease, n (\%) & $11(5.2)$ \\
HCC, n (\%) & $11(5.2)$ \\
Toxic (alcohol or drug-related), n (\%) & $13(6.2)$ \\
Cirrhosis, unknown etiology, n (\%) & $3(1.4)$ \\
Others, n (\%) & $9(4.3)$ \\
\hline
\end{tabular}

HCC, hepatocellular carcinoma

Table 2 Common pathogens of different periods after liver transplantation

\begin{tabular}{lcccc}
\hline Pathogen & $<$ 1 Month & 1-3 Months & >3 Months & Total \\
\hline Enterobacteriaceae & 28 & 10 & 3 & 41 \\
Acinetobacter baumanii & 27 & 0 & 0 & 27 \\
Pseudomonas aeruginosa & 19 & 2 & 0 & 21 \\
Strenotrophomonas maltophilia & 18 & 1 & 3 & 22 \\
Staphylococcus & 14 & 1 & 3 & 18 \\
Enterococcus & 10 & 2 & 2 & 14 \\
Cytomegalovirus & 9 & 3 & 0 & 12 \\
Fungus & 10 & 1 & 1 & 12 \\
\hline
\end{tabular}

Table 3 Risk factors of infection after liver transplantation

\begin{tabular}{|c|c|c|c|c|}
\hline Variables & With infection $(n=98)$ & Without infection $(n=112)$ & $P$ value & $P$ value-MA \\
\hline \multicolumn{5}{|l|}{ Preoperative variables } \\
\hline Age, mean (range) & $48.1(3-69)$ & $42.4(0.6-67)$ & 0.016 & \\
\hline Gender (Male), n (\%) & 78 (79.6) & 89 (79.5) & 1.000 & \\
\hline Diabetes, n (\%) & $22(22.4)$ & $18(16.1)$ & 0.291 & \\
\hline MELD or PELD before LT, mean (range) & $18.2(3-43)$ & $12.4(-9-46)$ & $<0.001$ & 0.002 \\
\hline Infection within 2 weeks before LT, $\mathrm{n}(\%)$ & $18(18.4)$ & $6(5.4)$ & 0.004 & \\
\hline Pneumonia before LT & $11(11.2)$ & $3(2.7)$ & 0.023 & \\
\hline Peritonitis before LT & $5(5.1)$ & $2(1.8)$ & 0.255 & \\
\hline \multicolumn{5}{|l|}{ Intraoperative variables } \\
\hline Duration of operation, mean (range), hours & $7.95(5-20.5)$ & $7.74(4.5-19)$ & 0.494 & \\
\hline Blood loss, mean (range), L & $2.3(0.2-22)$ & $1.1(0.1-5)$ & $<0.001$ & 0.002 \\
\hline Biliary anastomosis: Roux-en-Y, n (\%) & $4(4.1)$ & $11(9.8)$ & 0.178 & \\
\hline \multicolumn{5}{|l|}{ Postoperative variables } \\
\hline Immunosuppression with baxilimab, n (\%) & $12(12.2)$ & $15(13.4)$ & 0.839 & \\
\hline Reoperation, n (\%) & $10(10.2)$ & $4(3.6)$ & 0.093 & \\
\hline Rejection, n (\%) & $12(12.2)$ & $8(7.1)$ & 0.243 & \\
\hline Duration time of drainage tube, day & $29.6(3-282)$ & $12.8(1-66)$ & $<0.001$ & 0.009 \\
\hline
\end{tabular}

LT, liver transplantation; MELD, model for end-stage liver disease; PELD, pediatric end-stage liver disease; DCD, donation after cardiac death

Table 4 Univariate risk factors of mortality in 98 patients with postoperative infection 


\begin{tabular}{|c|c|c|c|c|c|c|}
\hline Variable & Survival $(n=82)$ & Death $(n=16)$ & $P$ value & $P$ value-MA & Odds ratio & 95\% confidence interval \\
\hline Age, mean (range) & $47.83(3-67)$ & $49.56(5-69)$ & 0.663 & & & \\
\hline Gender (Male), n (\%) & $68(82.9)$ & $10(62.5)$ & 0.088 & & & \\
\hline Infection within 2 weeks before LT, $n$ (\%) & $11(13.4)$ & $7(43.8)$ & 0.009 & 0.033 & 5.300 & 1.143-24.578 \\
\hline Pneumonia before LT & $6(7.3)$ & $5(31.2)$ & 0.016 & & & \\
\hline Peritonitis before LT & $2(2.4)$ & $3(18.8)$ & 0.030 & & & \\
\hline MELD or PELD before LT, mean (range) & $17.7(3-43)$ & $20.8(6-37)$ & 0.248 & & & \\
\hline MELD or PELD after 7 days of LT, mean (range) & $14.0(8-28)$ & $23.7(15-43)$ & 0.003 & 0.002 & 1.298 & $1.105-1.526$ \\
\hline Rejection, n (\%) & $12(14.6)$ & $0(0)$ & 0.206 & & & \\
\hline \multicolumn{7}{|l|}{ Complication after LT } \\
\hline eGFR<50ml/min after LT, n (\%) & $10(12.2)$ & $8(50.0)$ & 0.002 & & & \\
\hline Hepatic encephalopathy, n (\%) & $4(4.9)$ & $0(0)$ & 1.000 & & & \\
\hline
\end{tabular}

LT, liver transplantation; MA: Multivariate analysis; MELD, model for end-stage liver disease; PELD, pediatric end-stage liver disease

\section{Figures}

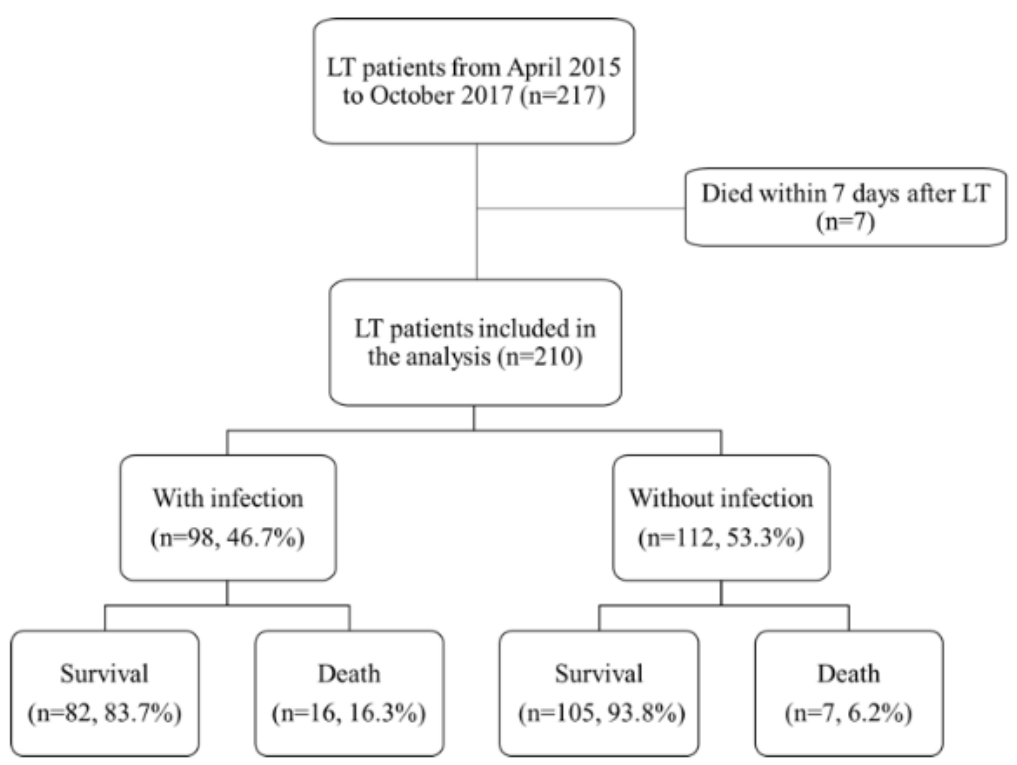

Figure 1

Flow chart of infections and survival after liver transplantation. LT, liver transplantation. 
- Acinetobacter baumanii

-Staphylococcus

- cytomegalovirus
Elebsiella pneumoniae a Pseudomonas aeruginosi afungus
EStrenotrophomonas maltophilia

Enterococcus faecium

mothers

Respiratory tract, $43.4 \%$

Abdominal, $21.4 \%$
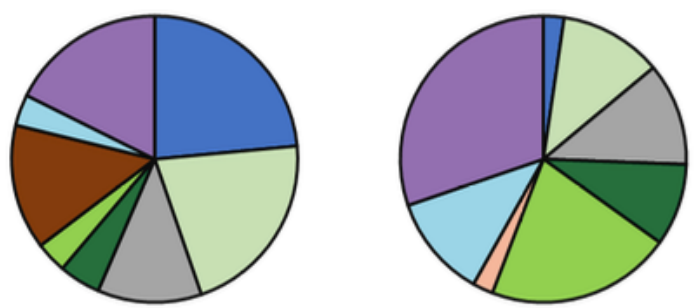

Biliary tract, $21.9 \%$

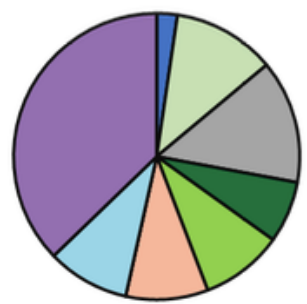

Bloodstream, 7.6\%

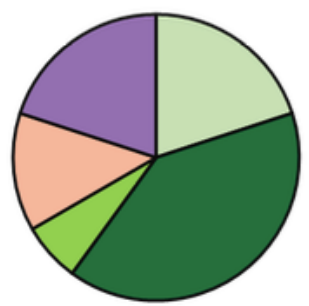

Figure 2

Frequency of different pathogens and sites of infections after liver transplantation.

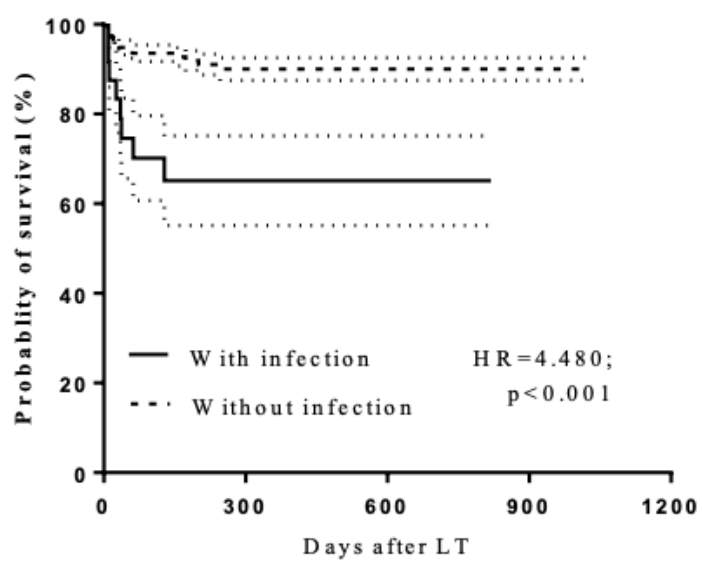

\section{Figure 3}

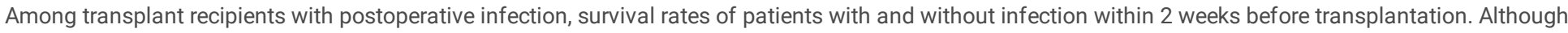

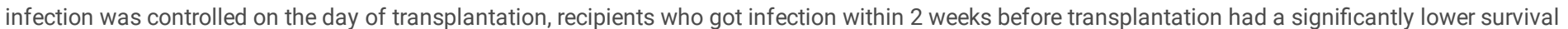

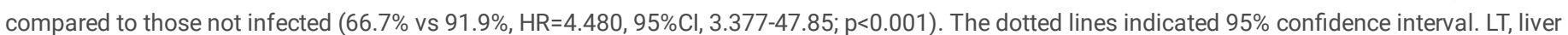
transplantation. 